\title{
Implementation of the Spur Involute Gear Model on Modelica
}

\author{
Ivan Kosenko Il'ya Gusev \\ Russian State University of Tourism and Service, Department of Engineering Mechanics \\ Cherkizovo-1, Moscow region, 141221, Russia
}

\begin{abstract}
A procedure to build up a dynamical model of the gearbox with spur involute mesh is being described. The main attention is paid to the design technology of the cylindrical bodies elastic contact models. To track geometry of contact implicit equations of algebraic/transcendental or differential-algebraic type are being used. At the same time dynamical models of the bodies involved, gearwheels and gearbox housing, continue to be three-dimensional. Analytical computational procedures to obtain gradients and Hessians are constructed for implementing the contact tracking algorithm for the involute guided cylindrical surfaces. The known Johnson model is applied for computing the contact elastic normal force. This force is defined as an implicit function of the mutual penetration depth at contact. Regular algorithm to compute the normal elastic force is built up. This algorithm is proved to be convergent. A detailed analysis of the virtual setup dynamic model is carried out.
\end{abstract}

Keywords: spur gear; involute; Johnson model; mesh properties; tracking algorithm

\section{Introduction}

Computer modeling and simulation of dynamics for gearboxes of different kinds is a wide spread engineering task. One might highlight here two extreme poles of approaches for models constructing. Firstly, the finite element method can be used for building up sufficiently detailed dynamical models. It is clear that the models created using such an approach consume quite significant amount of computational resources. Secondly, on the other pole of models range one might find simplified models of gearboxes dynamics allowing a very fast models for machines and their units to develop. Examples of such models are presented, for instance, in the Modelica Standard Library. In addition, there exist well developed models taking into account friction forces during the mesh processes in gearboxes [1]. One might also find several other interesting examples of the machinery applications including gearboxes models on Modelica [2, 3].

It is important to us to consider models incorporating both the rigid body dynamics sufficiently effective from the computational viewpoint and more detailed mesh models with different types of compliant contacts between teeth of gearwheels. The simplest problem in this way is the spur involute gear model implementation.

\section{Cylindrical symmetry of 3D-bodies contact}

Staying in frame of the spatial multibody dynamics classes previously developed $[4,5]$ it is quite natural to use additional rigid body $C$ playing the role of platform, for implementing a relative planar motion of the bodies, two gearwheels denoted as $A$ and $B$ in our case. These bodies assumed to have cylindrical shapes and are able to move in the plane orthogonal to their generatrix. Let $O_{C} x y z$ be a coordinate system rigidly connected with the body $C$, and for definiteness let $O_{C}$ be its center of mass. Assume the generatrix is always collinear to the axis $O_{C} z$ which can be expressed by the geometrical condition $\mathbf{k}_{\alpha}=\mathbf{k}_{C}(\alpha=A, B)$, where $\mathbf{k}_{\alpha}$ are the axis $O_{\alpha} z_{\alpha}$ unit vectors and $\mathbf{k}_{C}$ is the unit vector of the body $C$ axis $O_{C} z$. To keep bodies' motion parallel to the coordinate plane $O_{C} x y$ one has to require two algebraic conditions for the bodies $A$ and $B$ mass centers $z$-coordinates: $z_{O_{A}}=$ const, $z_{O_{B}}=$ const to be satisfied. All coordinates are assumed with respect to (w.r.t.) the frame $O_{C} x y z$.

Algebraic equations mentioned can be easily implemented in implicit form if one uses, for instance, constraints of the joint type [4] to fix the bodies $A$ and $B$ in the body $C$. In this case, the body $C$ itself can perform arbitrary spatial motions. We consider its movement as being convective in compound motions of the bodies $A$ and $B$ w.r.t. certain inertial frame of reference. Thus it is quite natural to call the body $C$ as a gear 
housing, and the bodies $A$ and $B$ are supposed to play the role of gearwheels.

One might build up mechanical tools for the cylindrical bodies contact using 2D-geometry techniques with aid of the above reduction to the plane $O_{C} x y$. For instance, the cylinders contact tracking model might be written, similarly to [6], in the form of six, algebraic or transcendental, equations as follows

$$
\begin{aligned}
\operatorname{grad} g_{A}\left(\mathbf{r}_{P_{A}}\right)= & \lambda \operatorname{grad} g_{B}\left(\mathbf{r}_{P_{B}}\right), \\
\mathbf{r}_{P_{A}}-\mathbf{r}_{P_{B}}= & \mu \operatorname{grad} g_{B}\left(\mathbf{r}_{P_{B}}\right), \\
g_{A}\left(\mathbf{r}_{P_{A}}\right)=0, \quad & g_{B}\left(\mathbf{r}_{P_{B}}\right)=0,
\end{aligned}
$$

where $\mathbf{r}_{P_{\alpha}}=\left(x_{P_{\alpha}}, y_{P_{\alpha}}\right)^{T}(\alpha=A, B)$ are the radius vectors for the points $P_{A}, P_{B}$ under tracking w.r.t. the housing coordinate system $O_{C} x y$; functions $g_{\alpha}(\mathbf{r})$ express equations for the curves bounding planar figures of the bodies $A$ and $B$ w.r.t. the axes $O_{C} x y ; \lambda, \mu$ are the auxiliary scalar variables. Totally the system (1) has six scalar equations w.r.t. six scalar variables $x_{P_{A}}, y_{P_{A}}, x_{P_{B}}$, $y_{P_{B}}, \lambda, \mu$.

To complete classes corresponding to models of contact one has to define contacting curves in the bodies own planar coordinate systems $O_{\alpha} x_{\alpha} y_{\alpha}$ in the form $f_{\alpha}\left(x_{\alpha}, y_{\alpha}\right)=0$. If $T_{\alpha}$ is an orthogonal $2 \times 2$-matrix defining current orientation of the body $\alpha$ planar figure then obviously the relations $g_{\alpha}(\mathbf{r})=f_{\alpha}\left[T_{\alpha}^{T}\left(\mathbf{r}-\mathbf{r}_{O_{\alpha}}\right)\right]$, $\operatorname{grad} g_{\alpha}(\mathbf{r})=T_{\alpha} \operatorname{grad} f_{\alpha}\left[T_{\alpha}^{T}\left(\mathbf{r}-\mathbf{r}_{O_{\alpha}}\right)\right]$ ought to take place.

Similarly, following the paper [7] one might easily construct a model to track the cylindrical contact by introducing a system of differential-algebraic equations of the form

$$
\begin{aligned}
\dot{\mathbf{r}}_{P_{A}}=\mathbf{u}_{P_{A}}, \quad \dot{\mathbf{r}}_{P_{B}}=\mathbf{u}_{P_{B}}, \quad \dot{\lambda}=\xi, \quad \dot{\mu}=\eta, & \\
{\left[\boldsymbol{\omega}_{A}, \operatorname{grad} g_{A}\right]+T_{A} \operatorname{Hess} f_{A} T_{A}^{T}\left(\mathbf{u}_{P_{A}}-\mathbf{v}_{P_{A}}\right)-} & \xi_{\operatorname{grad} g_{B}-} \\
\lambda\left(\left[\boldsymbol{\omega}_{B}, \operatorname{grad} g_{B}\right]+T_{B} \operatorname{Hess} f_{B} T_{B}^{T}\left(\mathbf{u}_{P_{B}}-\mathbf{v}_{P_{B}}\right)\right) & =\mathbf{0}, \\
\mathbf{u}_{P_{A}}-\mathbf{u}_{P_{B}}-\eta \operatorname{grad} g_{B}- & \\
\mu\left(\left[\boldsymbol{\omega}_{B}, \operatorname{grad} g_{B}\right]+T_{B} \operatorname{Hess} f_{B} T_{B}^{T}\left(\mathbf{u}_{P_{B}}-\mathbf{v}_{P_{B}}\right)\right) & =\mathbf{0}, \\
\left(\operatorname{grad} g_{A}, \mathbf{u}_{P_{A}}\right)-\left(\operatorname{grad} f_{A}, T_{A}^{T} \mathbf{v}_{P_{A}}\right) & =0, \\
\left(\operatorname{grad} g_{B}, \mathbf{u}_{P_{B}}\right)-\left(\operatorname{grad} f_{B}, T_{B}^{T} \mathbf{v}_{P_{B}}\right) & =0,
\end{aligned}
$$

where the vectors $\mathbf{v}_{P_{A}}, \mathbf{v}_{P_{B}}$ are relative, w. r. t. the body $C$, velocities of the bodies physical points currently located at the geometrical points $P_{A}, P_{B}$. One might calculate them according to the Euler formula

$$
\mathbf{v}_{P_{\alpha}}=\mathbf{v}_{O_{\alpha}}+\left[\boldsymbol{\omega}_{\alpha}, \mathbf{r}_{P_{\alpha}}-\mathbf{r}_{O_{\alpha}}\right] \quad(\alpha=A, B),
$$

where $O_{A}, O_{B}$ are the bodies mass-centers mentioned above, $\boldsymbol{\omega}_{A}, \boldsymbol{\omega}_{B}$ are relative w. r. t. the housing angular velocities of the bodies, always directed along the
$O_{C} z$-axis. Note that the points $O_{A}, O_{B}$ might be located at different levels of the $O_{C} z$-axis of the gearbox housing. But nevertheless one should regard the equations (2), (3) in their planar version as being projected onto the plane $O_{C} x y$.

In this case, for complete implementation of the contact model, we need to compute the gradients $\operatorname{grad} f_{\alpha}$ and Hessians Hess $f_{\alpha}$ at opposing points $P_{\alpha}$ in bodies own coordinates.

\section{Geometry of the spur involute gear}

One has to consider the involute equation in the plane $O_{\alpha} x_{\alpha} y_{\alpha}$ of the gearwheel coordinate system $O_{\alpha} x_{\alpha} y_{\alpha} z_{\alpha}$ for resolving the problem which has been formulated above for the case of the spur involute meshing. For this one has to apply polar coordinates $R_{\alpha}, \theta_{\alpha}$ defined for each body $\alpha$ in the following known way: $x_{\alpha}=$ $R_{\alpha} \cos \theta_{\alpha}, y_{\alpha}=R_{\alpha} \sin \theta_{\alpha}$. For the involute unwinding counterclockwise an equation for the polar coordinates can be deduced from the known relations [8] in the form

$$
\frac{\sqrt{R_{\alpha}^{2}-r_{\alpha b}^{2}}}{r_{\alpha b}}-\arccos \frac{r_{\alpha b}}{R_{\alpha}}-\theta_{\alpha}=0,
$$

where $r_{\alpha b}$ is the involute base circle radius. To compute grad $f_{\alpha}$ and Hess $f_{\alpha}$ one has to use formulae of the transformation $\left(x_{\alpha}, y_{\alpha}\right) \mapsto\left(R_{\alpha}, \theta_{\alpha}\right)$ and apply an auxiliary Jacobi matrices arising in the process of analytical calculations.

The contact tracking algorithm developed requires equation of a curve in the form $f(x, y)=0$ instead of equation (4). Introducing the notation

$$
p(R, \theta)=\frac{\sqrt{R^{2}-r_{b}^{2}}}{r_{b}}-\arccos \frac{r_{b}}{R}-\theta
$$

one can see easily that

$$
p(R, \theta)=f(R \cos \theta, R \sin \theta) .
$$

This equation is a starting point for producing all the formulae for gradients and Hessians. Indeed by virtue of (6) we have

$$
\operatorname{grad} p=\left(f_{x}, f_{y}\right)\left(\begin{array}{cc}
x_{R} & x_{\theta} \\
y_{R} & y_{\theta}
\end{array}\right)=\operatorname{grad} f \frac{\partial(x, y)}{\partial(R, \theta)}
$$

since $\operatorname{grad} p=\left(p_{R}, p_{\theta}\right)$. Therefore

$$
\operatorname{grad} f=\operatorname{grad} p\left[\frac{\partial(x, y)}{\partial(R, \theta)}\right]^{-1} .
$$


One can see from (5) that

$$
\operatorname{grad} p=\left(\frac{\sqrt{R^{2}-r_{b}^{2}}}{r_{b} R},-1\right)
$$

and from the polar coordinates definition the relation

$$
\frac{\partial(x, y)}{\partial(R, \theta)}=\left(\begin{array}{cc}
\cos \theta & -R \sin \theta \\
\sin \theta & R \cos \theta
\end{array}\right)
$$

follows. Hence,

$$
\left[\frac{\partial(x, y)}{\partial(R, \theta)}\right]^{-1}=\left(\begin{array}{cc}
\cos \theta & \sin \theta \\
-\frac{\sin \theta}{R} & \frac{\cos \theta}{R}
\end{array}\right) .
$$

To complete the computation of $\operatorname{grad} f$ as a function of $x, y$ one can remark that

$$
R=\sqrt{x^{2}+y^{2}}, \quad \cos \theta=\frac{x}{R(x, y)}, \quad \sin \theta=\frac{y}{R(x, y)}
$$

From (7) for the Hessian computation note that

$$
\begin{aligned}
\operatorname{Hess} p= & \left(\frac{\partial(x, y)}{\partial(R, \theta)}\right)^{T} \operatorname{Hess} f \frac{\partial(x, y)}{\partial(R, \theta)}+ \\
& f_{x} \operatorname{Hess} x+f_{y} \operatorname{Hess} y,
\end{aligned}
$$

where the following notation has been used

$$
\begin{aligned}
& \text { Hess } p=\left(\begin{array}{ll}
p_{R R} & p_{R \theta} \\
p_{\theta R} & p_{\theta \theta}
\end{array}\right)=\left(\begin{array}{cc}
\frac{r_{b}}{R^{2} \sqrt{R^{2}-r_{b}^{2}}} & 0 \\
0 & 0
\end{array}\right), \\
& \text { Hess } x=\left(\begin{array}{ll}
x_{R R} & x_{R \theta} \\
x_{\theta R} & x_{\theta \theta}
\end{array}\right)=\left(\begin{array}{cc}
0 & -\sin \theta \\
-\sin \theta & -R \cos \theta
\end{array}\right), \\
& \operatorname{Hess} y=\left(\begin{array}{ll}
y_{R R} & y_{R \theta} \\
y_{\theta R} & y_{\theta \theta}
\end{array}\right)=\left(\begin{array}{cc}
0 & \cos \theta \\
\cos \theta & -R \sin \theta
\end{array}\right),
\end{aligned}
$$

Now one can obtain from equation (12) a formula for the Hessian we sought

$$
\begin{aligned}
& \operatorname{Hess} f=\left[\left(\frac{\partial(x, y)}{\partial(R, \theta)}\right)^{T}\right]^{-1} \times \\
& \left(\operatorname{Hess} p-f_{x} \operatorname{Hess} x-f_{y} \operatorname{Hess} y\right)\left(\frac{\partial(x, y)}{\partial(R, \theta)}\right)^{-1} .
\end{aligned}
$$

All the objects included here on the right hand side have explicit expressions in polar coordinates. It is evident, these expressions can be resolved w. r. t. coordinates $x, y$ with aid of transformation (11).

\section{Contact force model}

The geometrical properties have been implemented as a class parameter for the base template described in [5] for the model outlined above with contact of involutes. Along with the geometrical properties model, the contact elastic normal force model plays a key role as well. According to the results of [9] the Johnson contact model [10] seems to be the most acceptable one for the case of the cylindrical bodies contact. The model can be expressed as an equation written for the case of so-called external contact

$$
h=\frac{N}{\pi E^{\star}}\left[\ln \frac{4 \pi E^{\star}\left(\rho_{A}+\rho_{B}\right)}{N}-1\right],
$$

representing an implicit function $N(h)$ for the specific normal elastic force, per unit of length along the cylinder generatrix, depending on the depth $h$ of mutual approach (penetration). Here in equation (13) $E^{\star}$ is the composite modulus of elasticity for the contact. It satisfies the equation

$$
\frac{1}{E^{\star}}=\frac{1-v_{A}^{2}}{E_{A}}+\frac{1-v_{B}^{2}}{E_{B}},
$$

where $E_{A}, E_{B}$ are Young's moduli of bodies' material, $v_{A}, v_{B}$ are Poisson ratios. Values $\rho_{A}, \rho_{B}$ are radii of curvature for involutes in the mesh each computed at current positions of the points $P_{A}, P_{B}$ respectively.

Remark 1 Staying in frame of the Hertz model conditions, we assume the contact area dimensions small as compared with the sizes of contacting bodies. Thus, the cylindrical involute surfaces in vicinities of points $P_{A}, P_{B}$ are approximated by the circular cylinders with an accuracy of order higher than two. Application of equation (13) means that we virtually replace the cylindrical surfaces with involutes as guides by the circular cylinders with the same radii of curvature at any current instant of simulation time. Evidently these cylindrical surfaces, involutive and circular ones, have mutual tangency of the second order.

Computational implementation of formula (13) inversion reduces to an equation w.r.t. dimensionless variables $x, y$ defined in the following way:

$$
x=\frac{e}{4\left(\rho_{A}+\rho_{B}\right)} h, \quad y=\frac{e}{4 \pi E^{\star}\left(\rho_{A}+\rho_{B}\right)} N .
$$

Then (13) becomes equivalent to the equation

$$
y \cdot \ln y=-x
$$

defining the implicit function $y(x)$. 
To resolve equation (14) correctly in vicinity of its evident solution $x=0, y=0$ one has to find a segment of monotonicity of the left hand side of (14). The zero solution corresponds to the case of beginning of the contacting process. In this case, a contact patch, generically rectangular area, degenerates into the 1Dline segment along the cylindrical surfaces generatrix. It is easy to see that the segment sought is $\left[0, e^{-1}\right]$. Within this segment the left hand side function of (14) decreases monotonically from zero to $-e^{-1}$. Thus one can define an area of applicability of the Johnson model by the following inequalities

$$
h \leq \frac{4\left(\rho_{A}+\rho_{B}\right)}{e^{2}}, \quad N \leq \frac{4 \pi E^{\star}\left(\rho_{A}+\rho_{B}\right)}{e^{2}} .
$$

From the involute properties and using Figure 1 the relation $\rho_{A}+\rho_{B}=\left|K_{A} K_{B}\right|$ is satisfied to within the (small enough) value $h$. For this reason, if the material stiffness is sufficiently large then the depth $h$ is small enough, and then the left condition of (15) is always satisfied. Here, the points $K_{A}, K_{B}$ are the points of a tangency between the line of action and base circles of gearwheels.

For real materials even the condition $h \ll\left|K_{A} K_{B}\right|$ is satisfied. Then for Young's moduli large enough the Johnson model is surely valid on the segment $y \in\left[0, e^{-1}\right]$ of monotonicity for the left side of equation (14). Since the derivative for the left side of equation (14) at $y=e^{-1}$ is equal to zero then we may furthermore restrict ourselves by set of strict monotonicity corresponding to the condition $0 \leq x<e^{-1}$, or equivalently to $0 \leq y<e^{-1}$.

Moreover, equation (14) has a singularity at zero. Therefore, we shall construct an algorithm for computing the function $y(x)$ taking into account that $0<$ $x<e^{-1}$. To proceed with the algorithm replace an unknown function $y(x)$ in equation (14) by the function $\eta(x)$ according to the formula $y(x)=x \eta(x)$. Then a new equation has the form

$$
\eta(\ln x+\ln \eta)+1=0
$$

Let us introduce here a new known independent variable $\mu$ instead of the old one $x$ according to the equation

$$
v=-\frac{1}{\ln x}
$$

The value $v$ is small and positive if the value $x$ is small and positive. Then equation (16) is transformed to the form

$$
\eta=v(1+\eta \ln \eta)
$$

more suitable for investigating and computing the solution for the given value of $v$.

To overcome the problem we need in an algorithm with the behavior regular enough in vicinity of zero. It turned out equation (17) delivers also an iteration process in the explicit form

$$
\eta_{n+1}=\mathrm{v}\left(1+\eta_{n} \ln \eta_{n}\right), \quad n=0,1, \ldots
$$

One might set any value $\eta_{0}$ satisfying the condition $0<\eta_{0} \leq e^{-1}$ as a guess value of iteration process (18). It is easy to see the numeric sequence $\left\{\eta_{n}\right\}_{n=0}^{\infty}$ built up using process (18) is strictly positive and bounded: $0<\eta_{n}<v$. Therefore, this sequence has at least one limit point $\eta_{\star}$. It is equivalent to an existence of the subsequence $\left\{\eta_{n_{k}}\right\}_{k=0}^{\infty}$ converging to this limit $\eta_{n_{k}} \longrightarrow$ $\eta_{\star}$ as $k \longrightarrow \infty$.

This limit is unique. Indeed, by virtue of (18) the limit satisfies equation (17). If there would be another different limit $\eta_{\star \star}$ then equation (17) should have at least two different solutions on the set $[0,1)$. Then as a consequence equation (14) should have two different solutions on the set $\left[0, e^{-1}\right)$ what is impossible because it has exactly one solution on this set.

Computations show that iteration process (18) converges fast enough. Merit of the process is that it works equally well for all admissible values of $x$. If $x$ becomes close to zero then the value $v>0$ is also small. Besides, for any arbitrarily small $\eta>0$ the function $\eta \ln \eta$ always stays uniformly bounded. Thus the iteration operator conserves its regularity for any admissible $x$.

Other class parameters of the contact model template in our case are following: (a) normal viscous term was selected similar to the implementation described in [7]; (b) tangent friction force model for definiteness and simplicity, similar, for example, to the paper [11], was selected as a regularized Coulomb friction law having a shape of the piecewise linear function of relative velocity at contact [6]. There are no difficulties for changing the corresponding class parameter and for applying any different model, more complicated than ones mentioned above.

\section{Algorithm of teeth pairs switching}

To describe the algorithm we assume gearwheels in rotational motion each such that the pinion, wheel $A$, rotates clockwise, and the gear $(B)$ does it counterclockwise. We consider a process of initial data generation later. When boosting the pinion tooth in generic case starts to penetrate the corresponding gear tooth 


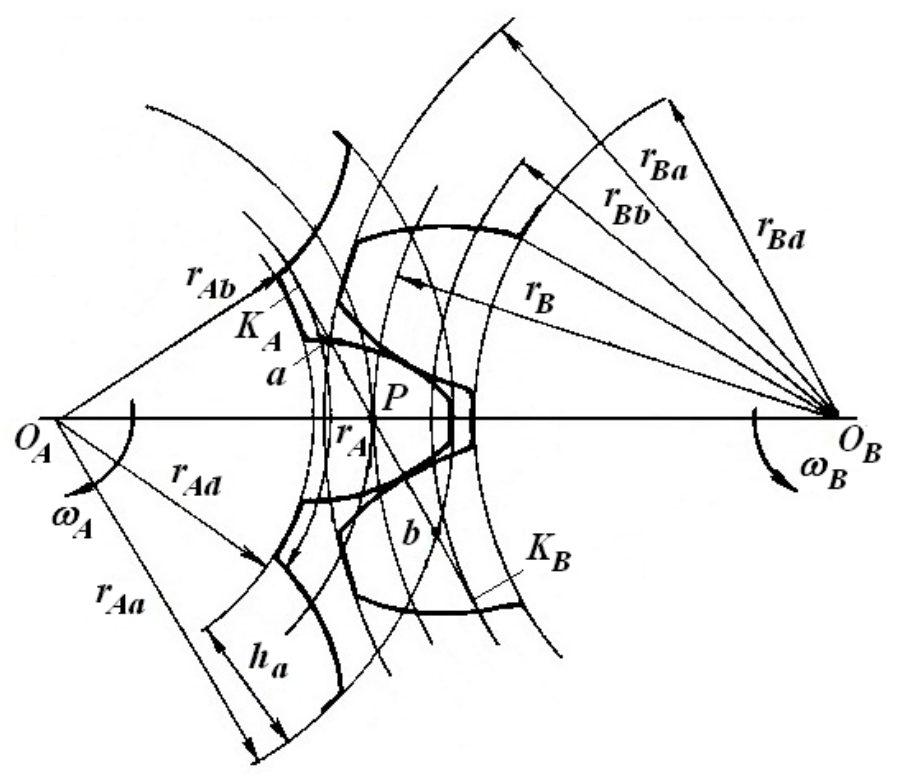

Figure 1: The mesh scheme

because of the compliance at contact. Suppose for simplicity this penetration continues during the motion without any backlash. The backlash possibility requires additional complications of the model.

Remind the points $P_{A}$ and $P_{B}$ of involutes are opposing each other at contact. Then using known properties of an involute one can prove the following

Assertion 1 The points $P_{A}$ and $P_{B}$ always lie on the line of action $K_{A} K_{B}$, see Figure 1.

Generally, due to the compliance of contact the point $P_{B}$ will be located on the line $K_{A} K_{B}$ to left from the point $P_{A}$ and above it, see Figure 1. Thus while the pinion rotates by a pitch angle $\Delta \gamma_{A}$, and the point $P_{A}$ reaches the position $b$ (starting from its initial position $a$ of the mesh cycle), contact itself does not really vanish. This may happen only after the point $P_{B}$ had also passed the position $b$.

On time interval, while the geometrical point $b$ had been passed initially by the point $P_{A}$ and then by $P_{B}$, contact model has to be more complicated than the Johnson one being applied here. This model strongly depends on the tooth tip relief had been implemented in the gearbox. The tip may be assumed sharp, as a point of the involute and addendum circle intersection, or curved with smoothed edges. For instance, it may have circular profile of the radius small enough between the involute and the addendum circle.

Exactly at the instance of the point $P_{A}$ passing through the position $b$, the similar point $P_{A}$ of the next teeth pair, denote it by prime $P_{A}^{\prime}$, passes through the position $a$. Really to this time, contact between the next teeth pair may already be existed. But requirements of the Johnson model will be implemented only after the point $P_{B}^{\prime}$ of the next pair contact will pass through the position $a$.

Thus in case of the compliant contact one cannot avoid a presence of two simultaneous contacts: (a) "decreasing" contact in vicinity of the position $b$ existing at this position after the point $P_{A}$ had passed through $b$; (b) "increasing" contact in vicinity of the position $a$ arising at $a$ before the point $P_{B}^{\prime}$ will pass through it. Remind that all these stages of transition arise simultaneously.

In our simplified approximate contact model we assume that exactly at the moment when the point $P_{A}$ passes through the position $b$ the Johnson contact model instantly switches from the point $b$ of the current teeth pair to the point $a$ of the next one. Exactly at this moment the point $P_{A}^{\prime}$ passes the position $a$. Denote this time instant as $t_{\star}$.

First of all one should set that

$$
\mathbf{r}_{P_{A}}\left(t_{\star}\right)=\mathbf{r}_{a} .
$$

To define the vector $\mathbf{r}_{P_{B}}\left(t_{\star}\right)$ it is sufficient to find a distance between points $P_{A}^{\prime}$ and $P_{B}^{\prime}$ of the new teeth pair. It is not difficult to check that this distance is equal exactly to the one between points $P_{A}$ and $P_{B}$ at the same instant. Note that simultaneously points $P_{A}^{\prime}$ and $P_{B}^{\prime}$ should lie on the line $K_{A} K_{B}$, first $P_{A}^{\prime}$ then $P_{B}^{\prime}$ if counting from $K_{A}$ to $K_{B}$. Thus one can also set that

$$
\mathbf{r}_{P_{B}}\left(t_{\star}\right)=\mathbf{r}_{P_{A}}\left(t_{\star}\right)+\mathbf{r}_{P_{B}}\left(t_{\star}-\right)-\mathbf{r}_{P_{A}}\left(t_{\star}-\right) \text {. }
$$

To obtain starting values $\lambda\left(t_{\star}\right), \mu\left(t_{\star}\right)$ for the next mesh cycle one has to compute norms of gradients 
$\left|\operatorname{grad} g_{A}\right|,\left|\operatorname{grad} g_{B}\right|$ (co)vectors and then use the first and the second equations of the system (1). Gradients are to be computed at points $P_{A}^{\prime}$ and $P_{B}^{\prime}$ respectively. Since we always suppose that gradients are directed "outside" the bodies at contact, we have to assume that

$$
\lambda\left(t_{\star}\right)<0, \quad \mu\left(t_{\star}\right)<0 .
$$

Furthermore, since functions $g_{A}, g_{B}$ are derived from the functions $f_{A}, f_{B}$ by translatory and rotary motions of the three dimensional space $\mathbf{R}^{3}$ then the following condition is satisfied

$$
\left|\operatorname{grad} g_{\alpha}\right|=\left|\operatorname{grad} f_{\alpha}\right| \quad(\alpha=A, B) .
$$

To compute the value $\left|\operatorname{grad} f_{\alpha}\right|$ let us apply equations (8), (9), (10) from above in the following way

$$
\begin{gathered}
\operatorname{grad} f_{\alpha}=\left(\frac{\sqrt{R_{\alpha}^{2}-r_{\alpha b}^{2}}}{r_{\alpha b} R_{\alpha}},-1\right)\left(\begin{array}{cc}
\cos \theta_{\alpha} & \sin \theta_{\alpha} \\
-\frac{\sin \theta_{\alpha}}{R_{\alpha}} & \frac{\cos \theta_{\alpha}}{R_{\alpha}}
\end{array}\right) \\
=\left(\frac{\sqrt{R_{\alpha}^{2}-r_{\alpha b}^{2}}}{r_{\alpha b} R_{\alpha}},-\frac{1}{R_{\alpha}}\right)\left(\begin{array}{cc}
\cos \theta_{\alpha} & \sin \theta_{\alpha} \\
-\sin \theta_{\alpha} & \cos \theta_{\alpha}
\end{array}\right),
\end{gathered}
$$

where $R_{\alpha}, \theta_{\alpha}$ are the polar coordinates in the body $\alpha$ coordinate system $O_{\alpha} x_{\alpha} y_{\alpha}$. One can see easily from last equation that the (co)vector $\operatorname{grad} f_{\alpha}$ is a result of the (co)vector

$$
\left(\frac{\sqrt{R_{\alpha}^{2}-r_{\alpha b}^{2}}}{r_{\alpha b} R_{\alpha}},-\frac{1}{R_{\alpha}}\right)
$$

rotation by the angle $\theta_{\alpha}$. Then one can write down the norm sought as

$$
\left|\operatorname{grad} g_{\alpha}\right|=\left|\operatorname{grad} f_{\alpha}\right|=\frac{1}{r_{\alpha b}} \quad(\alpha=A, B) .
$$

Thus the gradient (co)vector of the function defining the involute has a constant norm inversely proportional to the base circle radius. Now from the first and the second equations of the system (1) we have respectively

$$
\begin{aligned}
& \lambda\left(t_{\star}\right)=-\frac{\left|\operatorname{grad} g_{A}\right|}{\left|\operatorname{grad} g_{B}\right|}=-\frac{r_{B b}}{r_{A b}}, \\
& \mu\left(t_{\star}\right)=-\frac{\left|\mathbf{r}_{P_{B}}\left(t_{\star}-\right)-\mathbf{r}_{P_{A}}\left(t_{\star}-\right)\right|}{\left|\operatorname{grad} g_{B}\right|}= \\
& -r_{B b}\left|\mathbf{r}_{P_{B}}\left(t_{\star}-\right)-\mathbf{r}_{P_{A}}\left(t_{\star}-\right)\right| \text {. }
\end{aligned}
$$

And useful in all aspects result was obtained by the way:
Assertion 2 In case of involutes the equation

$$
\lambda=-\frac{r_{B b}}{r_{A b}}
$$

represents an integral of motion for the contact tracking system of DAEs (2), (3).

Indeed, one can see from above that the variable $\lambda$ keeps its initial value all time of simulation

$$
\lambda(t) \equiv \lambda\left(t_{0}\right)=\lambda\left(t_{\star}\right)=-\frac{r_{B b}}{r_{A b}} .
$$

This property may be very useful to control an accuracy of computations during the simulation process.

\section{Some details of implementation}

As it was already mentioned the template

ContactConstraintBaseTemplate

developed earlier [5] has been applied for implementing the contact model of two gearwheels with spur involute gearing. The template has four class parameters defining models of: (a) the normal elastic force, (b) the normal force of viscous resistance, (c) the tangent force of resistance for relative slipping at contact, (d) geometry of surfaces in vicinity of contact, in our case they are the cylinders guided by an involute.

As it was already noted we guess approximately that a contact patch is the rectangular strip, in general thin enough. The normal elastic force is assumed to be uniformly distributed in one dimension along the generatrix of cylinder over the patch.

Let us consider in more details an implementation of the class parameter responsible for geometry properties of the contact. This class is implemented as a four-level hierarchy of inheritance for the properties and behavior:

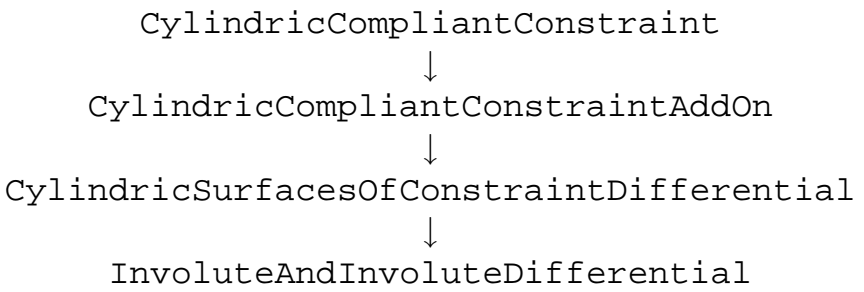

A differential-algebraic equations are applied here for implementing the contact tracking algorithm.

A base class for all geometry classes implementing cylindrical contact is the model CylindriccompliantConstraint. This class is responsible for computation of geometric and kinematic properties of the points $P_{A}$ and $P_{B}$ under tracking w. r. t. the third body $C$, the gearbox housing. These properties are described in particular by the following variables: 
- rAr, rBr are the points $P_{A}$ and $P_{B}$ relative positions;

- gradgAr, gradgBr are the cylindrical surfaces gradient vectors computed at points $P_{A}, P_{B}$ respectively in the frame of coordinates $O_{C} x y z$;

- normar is the unit vector normal to an outer surface of the body $A$ computed at the point $P_{A}$ w. r. t. the coordinate system $O_{C} x y z$;

- vrAr, vrBr are relative velocities of bodies' $A, B$ points currently occupying the geometric points $P_{A}, P_{B}$ locations;

Modelica code of the current class under description, its section of equations, reads

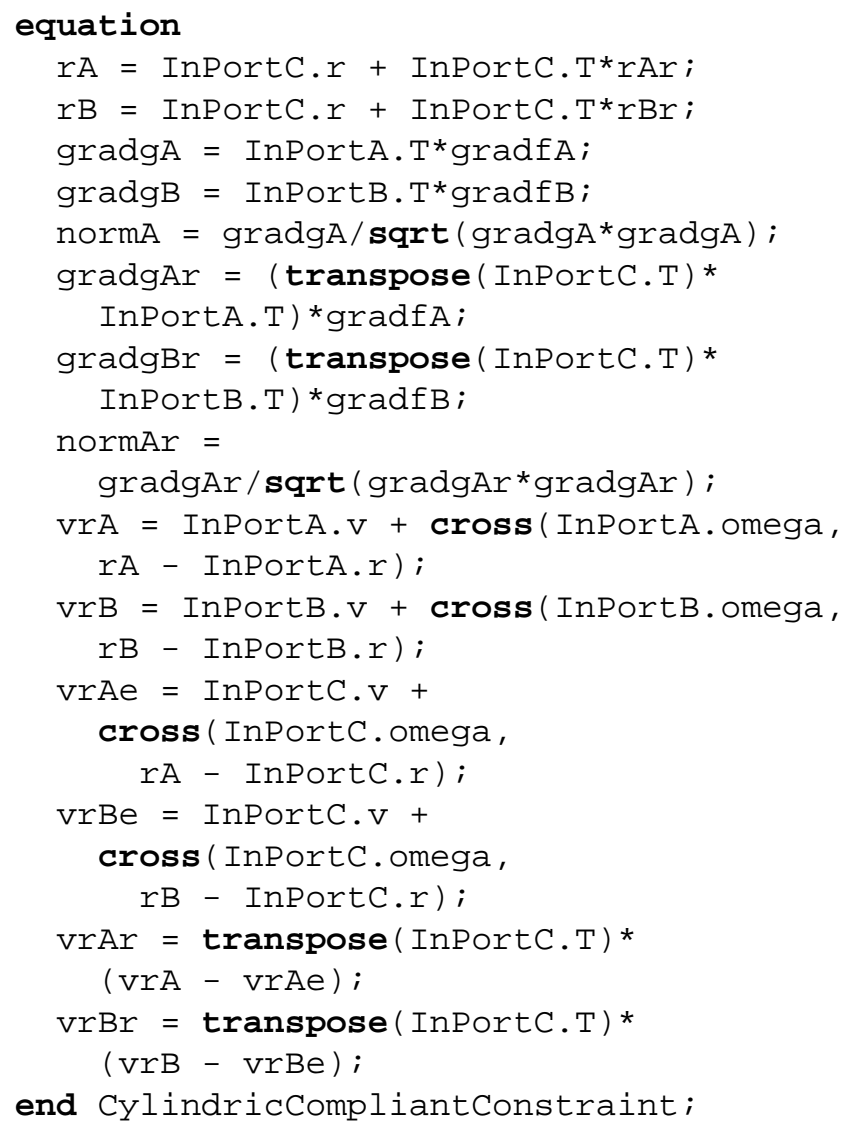

In the derived class CylindricCompliantConstraintAddOn:

- PA, PB are variables for coordinates of the points where a resultant contact forces are applied, in directions of bodies $A$ and $B$ respectively;

- relvnr is the normal component, in case of PA $=\mathrm{PB}$, of the velocity for the point $\mathrm{PA}$ of the body $A$ relative to the body $B$;

- relvtr is the tangent component of the the body $A$ relative velocity at $\mathrm{PA}=\mathrm{PB}$ w. $\mathrm{r}$. t. the coordinate system $O_{C} x y z$;
- kappa is the contact indicator which is: (a) equal to zero for the case of the surfaces touching each other by segment of strait line, (b) positive and equals to the distance between the surfaces for the case of contact absence, (c) negative and characterizes depth of mutual penetration for the case of contact, contact patch has a rectangular shape;

- kappaA, kappaB are the parameters defining elastic properties of bodies $A$ and $B$ respectively.

Section of equations/behavior for this model has the following code

\section{equation}

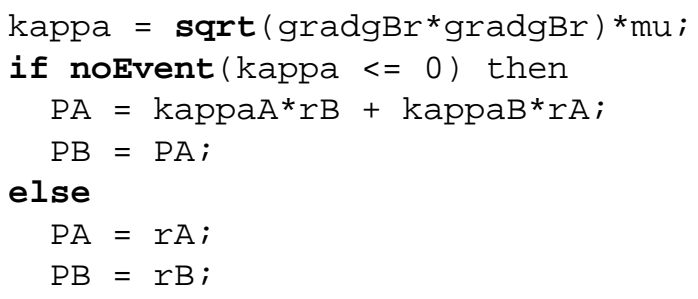

The next derived class CylindricSurfacesofConstraintDifferential implements the DAE system (2), (3). Its Modelica code is similar to one of the class SurfacesofConstraintDifferential described in [7] for the generic case of the Hertz-point model. The difference concerns an account of the cylindrical symmetry for the current case.

Finally, the contact surfaces, rather curves bounding planar figures of bodies, specifications are defined in the last class of the inheritance chain InvoluteAndInvoluteDifferential. This model implements an algorithm described in Section 3 for computing of 
gradients and Hessians in bodies' coordinates systems. As one can see from Section 3 corresponding Modelica code has to be bulky enough.

It is clear that when working two teeth of gearwheels $A$ and $B$ cannot stay in the meshing process during long time. One can see in Figure 1 that in case of the pinion rotation clockwise the contact point (segment of line), or rather contact patch small enough, moves from the point $a$ to the point $b$ of the line of action $K_{A} K_{B}$. At the very moment of contact loss for the current pair of teeth at the point $b$ the next pair arrives at contact, the point $a$, and new "point" of contact starts its motion along the meshing strait line of action.

One has to note here that for simplicity we consider a mesh process without overlapping of time intervals for the teeth pairs contacting. If they are overlapped then one should create at least two contact objects "connecting" the objects of bodies $A$ and $B$. These objects are to be activated/deactivated alternatively when arriving at/departing from the point $a / b$.

While the contact patch moves from its position $a$ to the position $b$ each of gearwheels $A, B$ rotates by the pitch angle $\Delta \gamma_{A}, \Delta \gamma_{B}$ respectively. The last class of the inheritance line considered above besides the computation of gradients and Hessians implements also a switching process for the pairs of gearwheels thus synchronizing this switching with the corresponding angles of rotation for the bodies $A$ and $B$.

This mechanism for discontinuous jumps of the contact points is implemented by the Modelica event handling facility. Code of the class InvoluteAndInvoluteDifferential fragment concerning required switching reads

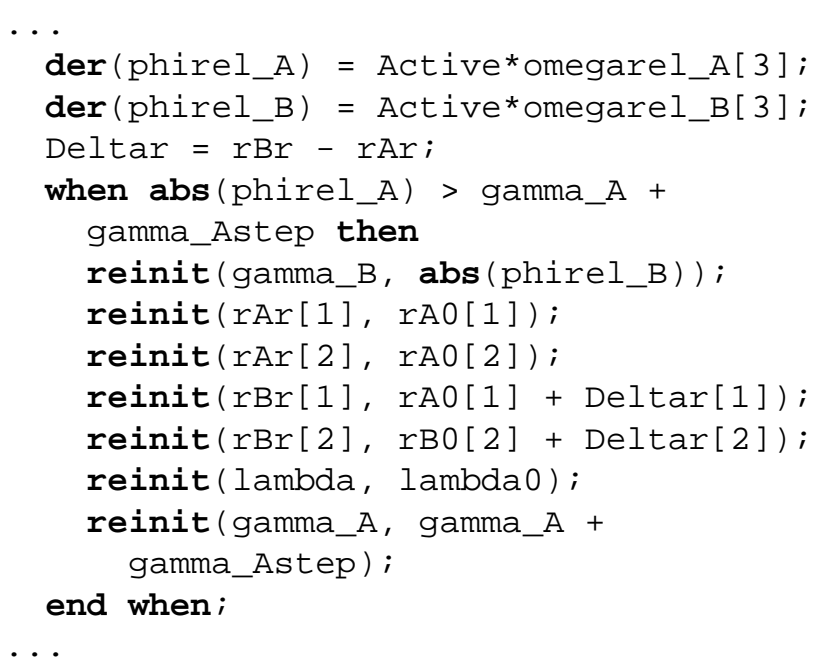

Here the variables phirel_A, phirel_B are to accumulate an angles of rotation for the bodies $A, B$ w. r. t. the housing $C$. They are the model state variables having derivatives defined as $z$-components of the wheels relative angular velocities vectors omegarel_A and omegarel_B.

In the code fragment above, variables $r A 0, r B 0$, in addition to ones already described, correspond to the points $P_{A}, P_{B}$ initial position, at the point $a$ in Figure 1, vectors in the contact tracking algorithm. Relative, w. r. t. $P_{A}$, position of the point $P_{B}$ for the new teeth pair being directed, as we know, along the line of action $K_{A} K_{B}$, has to be equal, because of rigidity, to the similar position for the previous pair losing contact at the event. This relative position is tracked by the variable Delta.

The variables mu, lambda correspond to the variables $\mu, \lambda$ in equations (2), (3); lambda 0 correspond to the $\lambda$ initial value at the position $a$; gamma_A, gamma_B are the variables for the gearwheels angles of rotation changing by the pitch values $\Delta \gamma_{A}, \Delta \gamma_{B}$ being stored in variables gamma_Astep, gamma_Bstep. Note that for correct handling of the switching process it is sufficient to track only the pinion, body $A$, angle of rotation and use only the variables gamma_A, gamma_Astep, phirel_A, omegarel_A. Initial depth of penetration, just after the contact switch, for the new pair is defined by the variable mu remains the same as for the previous pair of teeth in contact. This is because the gradient, from the right hand side of the second equation in system (1), norm stays constant in case of the involute. This constant is equal to the value $1 / r_{B b}$. For definiteness the wheel $A$ supposed to rotate monotonically clockwise.

\section{Computational experiments}

To perform a computational testing program for the gearbox model one builds up a virtual setup consisting of two gearwheels: the pinion $A$ and the driven gear $B$. For simplicity one assumes the gearbox housing $C$ be fixed w. r. t. inertial frame of reference, and the origin $O_{C}$ of its coordinate system $O_{C} x y z$ coincides with the pinion geometrical center $O_{A}$. Cylindrical revolute joint connecting the bodies $A$ and $C$ is also located at the point $O_{C}$. The gearwheel $B$ center $O_{B}$ is located on the horizontal axis $O_{C} x$. Here, at $O_{B}$, a cylindrical revolute joint connecting the body $B$ and the auxiliary slider $S$ is located. The slider $S$ is in turn able to slip freely w. r. t. the body $C$ along the axis $O_{C} x$, though this sliding performs with a resistance of the spring of high stiffness with the damper. This spring connects bodies $C$ and $S$ between one another.

We introduced in the current experimental setup un- 


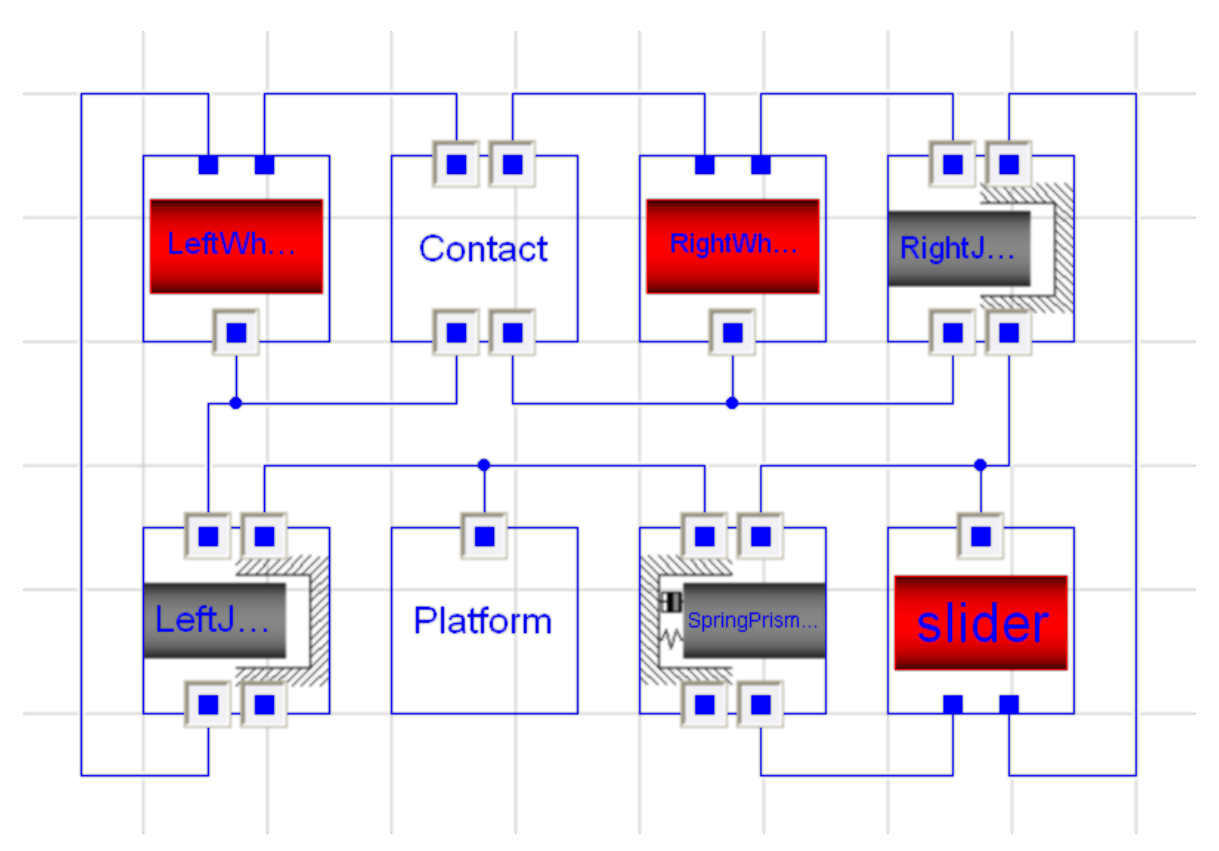

Figure 2: The Virtual Setup Visual Model

der description a compliance between the bodies $B$ and $C$. This compliance is implemented by the auxiliary slider mentioned and is directed along the line $O_{A} O_{B}$ connecting the wheels centers and lying on the axis $O_{C} x$. Such a construct prevents the static indefiniteness in the model for the case of rigid contact at the mesh point of gearwheels $A$ and $B$. A visual model of the setup is shown in Figure 2.

\subsection{Parameters of the model}

The following independent parameters are defined in the mesh model:

- $z_{A}=20$ is number of teeth for the pinion;

- $z_{A}=30$ is number of teeth for the driven gearwheel;

- $r_{A}=0.2 \mathrm{~m}$ is the pinion pitch circle radius.

After that the remaining geometry parameters of the mesh are computed as follows:

- $n=z_{B} / z_{A}$ is the transmission ratio;

- $r_{B}=n r_{A}$ is the pitch circle radius of the driven gearwheel;

- $\Delta \gamma_{A}=2 \pi / z_{A}, \Delta \gamma_{B}=2 \pi / z_{B}$ are the gearwheels angular pitches;

To define the mesh further it is essential to set the pressure angle $\alpha_{w}$ value. It can be chosen using the condition

$$
\alpha_{w}>\alpha_{w i n f}
$$

where $\alpha_{w \text { inf }}=\inf \alpha_{w}$ is the lower bound for all the pressure angles permissible by the parameters above. Its value is defined by the formula

$$
\alpha_{w \text { inf }}=\arctan \frac{2 \pi}{z_{A}(1+n)} .
$$

For definiteness we will use the following value

$$
\alpha_{w}=1.05 \alpha_{w \text { inf }} .
$$

Furthermore, using the pressure angle and transmission ratio one cam compute sequentially all geometrical parameters needed which are shown in Figure 1. First the base circles radii can be found as

$$
r_{\alpha b}=r_{\alpha} \cos \alpha_{w} \quad(\alpha=A, B) .
$$

Then one can compute a full length of the line of action as follows

$$
\left|\overrightarrow{K_{A} K_{B}}\right|=r_{A}(1+n) \sin \alpha_{w} .
$$

At the same time a length of any segment $[a, b]$ along this line is exactly the length of arc for any of the base circles corresponding to the pitch angle $\Delta \gamma_{A}$ or $\Delta \gamma_{B}$

$$
|\overrightarrow{a b}|=r_{\alpha} \Delta \gamma_{\alpha} \quad(\alpha=A, B) .
$$

Initial distance between the gearwheels centers is equal to the value $L=r_{A}+r_{B}$. To compute initial conditions for the contact tracking system of DAEs (2), (3) we need in additional computations. From the description above we have for absolute initial coordinates of the points $O_{C}$ and $O_{A}$

$$
\mathbf{r}_{O_{C}}=\mathbf{r}_{O_{A}}=(0,0,0)^{T} \text {. }
$$


Thus an initial position of the gear center is defined by the equation

$$
\mathbf{r}_{O_{B}}=(L, 0,0)^{T} .
$$

Initial positions of the points $K_{A}$ and $K_{B}$ can be computed easily, see Figure 1, with use of the following vector formulae

$$
\begin{aligned}
& \mathbf{r}_{K_{A}}=\mathbf{r}_{O_{A}}+r_{A b}\left(\cos \alpha_{w}, \sin \alpha_{w}, 0\right)^{T}, \\
& \mathbf{r}_{K_{B}}=\mathbf{r}_{O_{B}}-r_{B b}\left(\cos \alpha_{w}, \sin \alpha_{w}, 0\right)^{T} .
\end{aligned}
$$

After that the line of action directing vector can be obtained as $\overrightarrow{K_{A} K_{B}}=\mathbf{r}_{K_{B}}-\mathbf{r}_{K_{A}}$. And now one can define a position of the point $a$, where the contact process begins, in the form

$$
\mathbf{r}_{a}=\mathbf{r}_{K_{A}}+\frac{1}{2}\left(\left|\overrightarrow{K_{A} K_{B}}\right|-|\overrightarrow{a b}|\right) \frac{\overrightarrow{K_{A} K_{B}}}{\left|\overrightarrow{K_{A} K_{B}}\right|},
$$

and also an initial position of the point $b$, where the contact losses, has the representation

$$
\mathbf{r}_{b}=\mathbf{r}_{a}+\frac{|\overrightarrow{a b}|}{\left|\overrightarrow{K_{A} K_{B}}\right|} \overrightarrow{K_{A} K_{B}}
$$

After the endpoints $a, b$ of an active segment of the line of action have been defined it is time to compute the addendum radius $r_{B a}$ of the gear as a distance between the point $a$ and the initial position of $O_{B}$, see Figure 1. The addendum radius $r_{A a}$ of the pinion is in turn a distance between an initial position of the point $b$ and $O_{A}$. These radii are defined by the equations

$$
r_{A a}=\left|\mathbf{r}_{b}-\mathbf{r}_{O_{A}}\right|, \quad r_{B a}=\left|\mathbf{r}_{a}-\mathbf{r}_{O_{B}}\right| .
$$

To compute initial angles of rotation for the pinion and gear we assume that at an initial instant of simulation teeth of an initial pair are touching each other geometrically without any pressure, and, as a result, mutual penetration is absent. An initial angular velocities of the gearwheels assumed equal to zero. For definiteness we also assume that the axis $O_{A} x_{A}$ of the body $A$ crosses the base circle exactly at a root point of the involute. This involute defines a surface of the tooth contacting with its mate exactly at the point $a$. Similarly, the body $B$ axis $O_{B} x_{B}$ passes through the root point of the contact involute of the body $B$ at initial instant.

One can compute the polar angles of each the involute mentioned above using the equations (4) with the following equations $(\alpha=A, B)$

$$
\theta_{\alpha}=\frac{\sqrt{\left|\mathbf{r}_{a}-\mathbf{r}_{O_{\alpha}}\right|^{2}-r_{\alpha b}^{2}}}{r_{\alpha b}}-\arccos \frac{r_{\alpha b}}{\left|\mathbf{r}_{a}-\mathbf{r}_{O_{\alpha}}\right|},
$$

Thus we can define an initial values for the angles of rotation of bodies $A$ and $B$ in the form

$$
\varphi_{\alpha}\left(t_{0}\right)=\arg \zeta_{\alpha}-\theta_{\alpha} \quad(\alpha=A, B)
$$

where complex numbers $\zeta_{A}, \zeta_{B}$ are defined via the vectors $\mathbf{r}_{a}-\mathbf{r}_{O_{\alpha}}$ components as

$$
\zeta_{\alpha}=\left(x_{a}-x_{O_{\alpha}}\right)+i\left(y_{a}-y_{O_{\alpha}}\right) \quad(\alpha=A, B) .
$$

Note that the function arg of complex argument has a computer implementation as a standard library function atan2.

Initial quaternions of bodies $A$ and $B$ orientation are defined using known formulae

$\mathbf{q}_{\alpha}\left(t_{0}\right)=\left(\cos \frac{\varphi_{\alpha}\left(t_{0}\right)}{2}, 0,0, \sin \frac{\varphi_{\alpha}\left(t_{0}\right)}{2}\right)^{T}(\alpha=A, B)$.

State variables of the DAE system (2), (3) tracking contact are to satisfy the following initial conditions

$$
\mathbf{r}_{P_{A}}\left(t_{0}\right)=\mathbf{r}_{P_{B}}\left(t_{0}\right)=\mathbf{r}_{a}, \mu\left(t_{0}\right)=0, \lambda\left(t_{0}\right)=-\frac{r_{B b}}{r_{A b}} .
$$

Note here that in case of the involute the state variable $\lambda(t)$ turned out to be constant value

$$
\lambda(t) \equiv \text { const }=\lambda\left(t_{0}\right) .
$$

Thus this equation represents exactly integral of motion, and it can be used effectively to control an accuracy of computations.

Finally, in the example under consideration the constant driving torque $M_{A}=(0,0,-1 \mathrm{~N} \cdot \mathrm{m})^{T}$ assumed being applied to the pinion $A$ while the viscous torque of resistance $M_{B}=\left(0,0,-10 \dot{\varphi}_{B}\right)^{T}$ is applied to the gear $B$. Gearwheels themselves assumed made of steel with Young's modulus $E_{A}=E_{B}=2 \cdot 10^{11} \mathrm{~Pa}$ and Poisson ratio $v_{A}=v_{B}=0.3$, and have the same width, along the axis of rotation, of $0.1 \mathrm{~m}$.

\subsection{Dynamic transmission error}

A value of the dynamic transmission error (DTE) has been chosen for the computational verification. If force of friction exists at contact then DTE is not constant. First of all let us introduce the auxiliary variable

$$
\Delta=-r_{A b} \varphi_{A}-r_{B b} \varphi_{B} .
$$

This value characterizes a mismatch for the base circles arc lengths. If teeth in pairs contacting have an ideal "rigid" unilateral constraints without compliance, and switching between teeth pairs is also ideal then the value of $\Delta$ has to be an identical zero. 


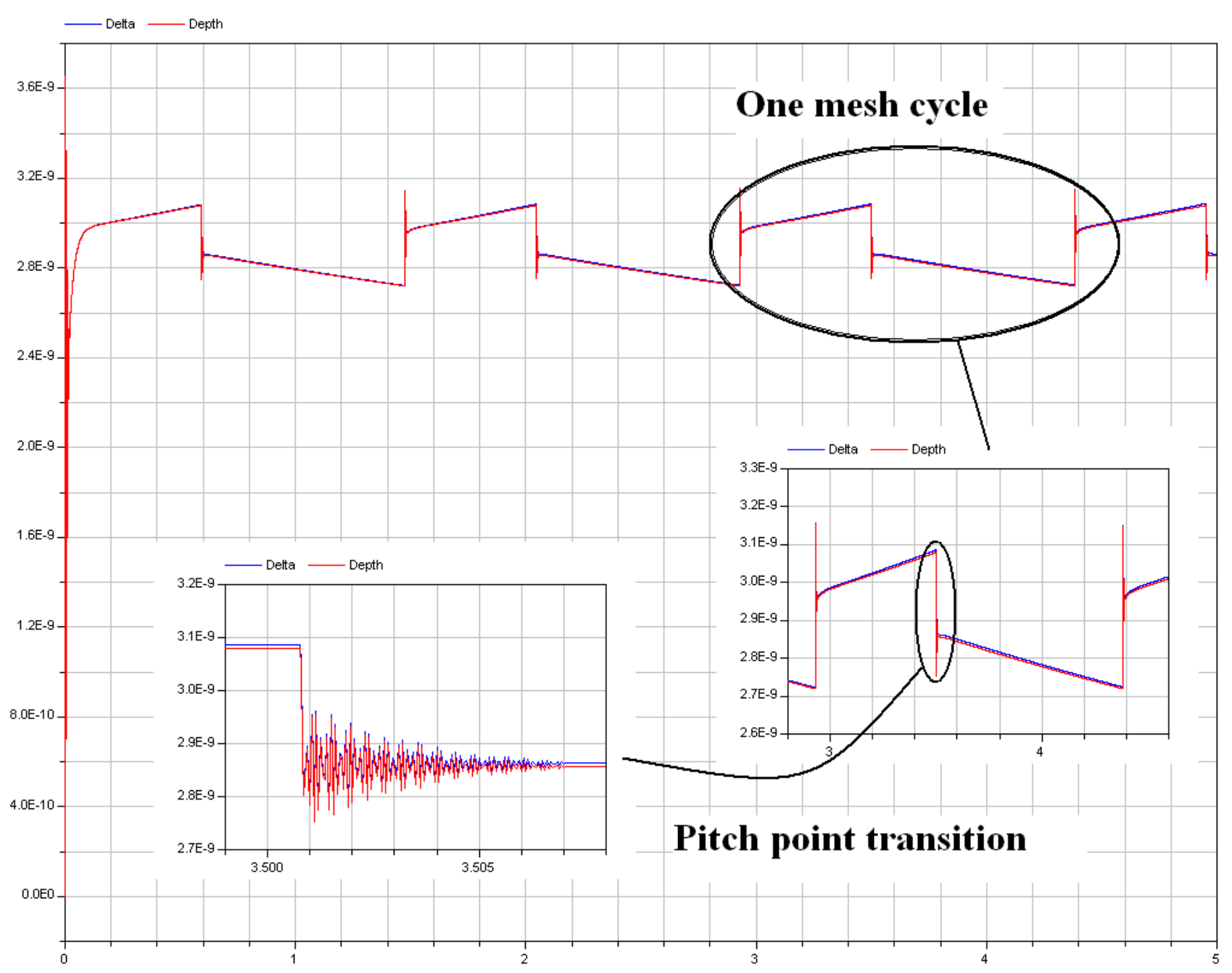

Figure 3: Comparison of the discrepancy (Delta) and the depth of penetration (Depth)

To undertake an analysis more detailed let us consider the results of numeric experiments. Firstly, one can remark that in case of the involute mesh a discrepancy $\Delta(t)$ from (19) has to be identical with the depth of mutual penetration of rigid teeth in vicinity of contact point. Indeed, the discrepancy (19) is exactly the difference between the arc distances of base circles while each of the wheels $A$ and $B$ rotates. This difference is accumulated from the very initial instant of simulation.

On the other hand it is known that the segment $P_{A} P_{B}$ is a perpendicular common to the teeth involutes penetrating each other, and simultaneously $P_{A} P_{B}$ lies exactly on the line $K_{A} K_{B}$ and its length is exactly the depth of teeth mutual penetration. Then there exists the only geometric possibility: the condition

$$
\left|\overrightarrow{P_{A} P_{B}}\right| \equiv \kappa(t) \equiv \Delta(t)
$$

has to be satisfied. The functions $\Delta(t), \kappa(t)$ derived independently in the model are compared in Figure 3.
An effect obtained in an angular displacements due to pressing and subsequent penetrating in the Johnson contact model is certainly like one derived due to torsional deformations of elastic gearwheels [12, 13]. Moreover, finite element modeling do not disturb in any essential degree the whole dynamical picture if used to simulate teeth bending when contacting [14].

Indeed, one can compute the DTE according to the formula

$$
\delta=-r_{A b} \psi_{A}-r_{B b} \psi_{B}
$$

similar to (19). Here the values $\psi_{A}, \psi_{B}$ are the angular displacements of the pinion and gear from their mean nominal positions $\Phi_{A}(t), \Phi_{B}(t)$ such that the following equations fulfill

$$
\varphi_{\alpha}(t)=\Phi_{\alpha}(t)+\psi_{\alpha}(t) \quad(\alpha=A, B)
$$

These nominal values $\Phi_{\alpha}(t)$ correspond just to the case of rigid contact satisfying evidently the following 
kinematic identity

$$
-r_{A b} \Phi_{A}(t)-r_{B b} \Phi_{B}(t) \equiv 0 .
$$

Hence, it turns out in frame of our considerations that the identity

$$
\Delta(t) \equiv \delta(t)
$$

takes place.

Let us investigate now sources of the DTE presented in Figure 3. If one eliminates completely in the model the friction between teeth surfaces then the value $\delta(t)$ will grow asymptotically to its limit value thus providing a systematic error of transmission, see Figure 4, blue curve. The reason for this error is evidently a mutual penetration of the compliant contact model of Johnson.

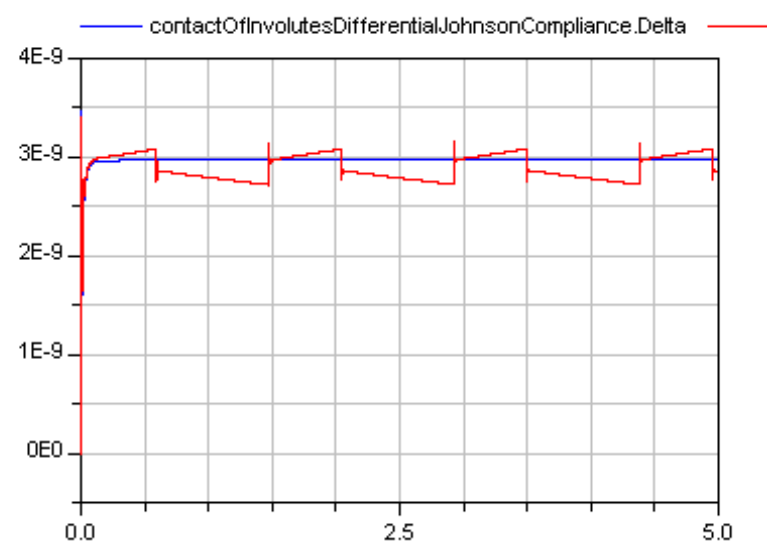

Figure 4: The DTE without (blue) and with (red) friction

If one introduces to the whole gearbox model, the simplest model of the Coulomb friction with the coefficient $f=0.3$ then the systematic error will be superimposed by the periodic one, see Figure 4, red curve. This latter error has discontinuities at instants of the contact teeth pair changes and at the instant when the contact patch passes through the pitch point $P$, see Figure 1 . The periodic DTE almost completely coincides with similar curves presented in papers $[12,13]$. In the graphs of these papers one can note only small variations from exact curves of our Figures 3,4. An origin of these variations is evidently additional small deviation derived due to more exact account of the elastic torsion oscillations considered in [12, 13]. Additional splash of weak torsional oscillations one can observe in $[12,13]$ exists because of the multiplicity of teeth contacts in that model: time segments overlap for the mesh cycles of the nearby pairs. So when contact of the previous teeth pair vanishes then additional elastic disturbance arises. Remind that for simplicity we
Table 1: Comparative efficiency

\begin{tabular}{|c|c|c|}
\hline $\begin{array}{c}\text { Type of the } \\
\text { contact model }\end{array}$ & $\begin{array}{c}\text { Coefficient } \\
\text { of friction }\end{array}$ & $\begin{array}{c}\text { CPU } \\
\text { time }\end{array}$ \\
\hline Johnson's & 0.3 & 20.4 \\
\hline Johnson's & 0 & 16.1 \\
\hline rigid & 0.3 & 13 \\
\hline rigid & 0 & 11.4 \\
\hline
\end{tabular}

consider in our model the case of mesh ratio which is equal to one.

\subsection{Comparison with a rigid contact}

A goal of our further numerical experiments is to compare two contact models when meshing: (a) the Johnson model, (b) the rigid contact model without compliance. The results of models under comparison simulation run showed for illustration in Figure 5. The normal contact force is counted along the $y$-axis of the plot. Case of the Johnson model corresponds to the blue curve while the red one is for the case of rigid contact model.

Comparison of the simulation results shows in Figure 5 that both contact models, the Johnson one and the rigid contact, bring the same dynamic result. The only difference is that the Johnson model generates additional oscillations of the normal contact force being superimposed on the normal force behavior for the rigid case. Moreover, one can see from detailing shown in Figure 5 that rigid contact model looks like a result of the procedure of averaging for the dynamics with the compliant contact, Johnson, model.

An advantage of the latter case is that this case of the contact model makes it possible to apply an arbitrary number of contacts for the body in the multibody system dynamics model without any restrictions. At the same time the rigid contact model does not allow such a possibility.

On the other hand, any contact model based on the FEM code application requires much more computational resources than in case of the "simple" compliant model analysed above. To compare an effectiveness of the Johnson contact model and the model using the rigid unilateral contact constraint consider Table 1 with preliminary relative estimations of the CPU time, in seconds, needed for both cases with addition of the friction force influence. Here the results of the simulation run are presented, without any optimization, for the model time of 5 seconds. 


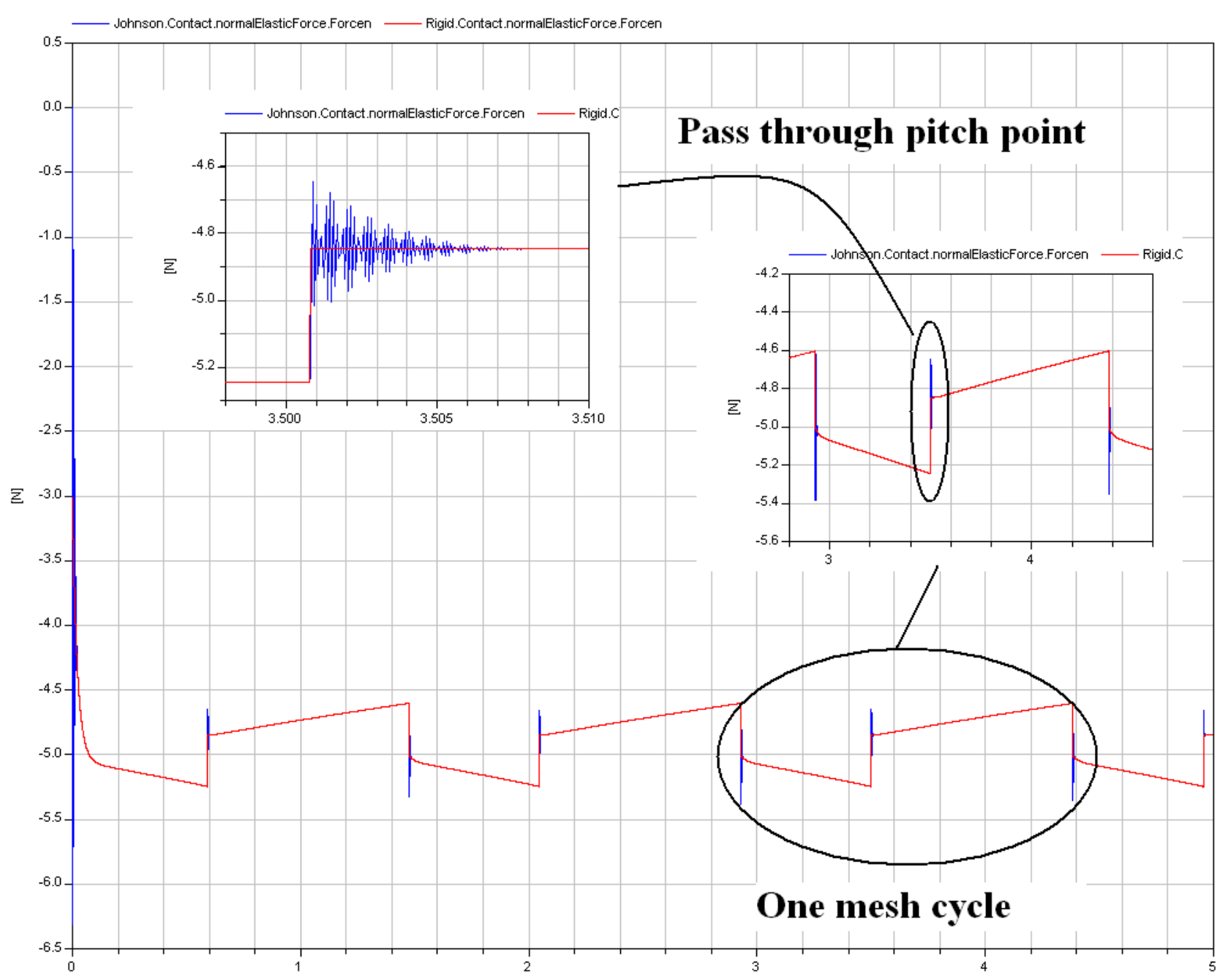

Figure 5: Comparison of the models with (blue) and without (red) compliance at contact

One can note easily from Table 1 that the Johnson model increases computational time in some degree, not larger than twice, in compare with the fastest case of the rigid contact. And simultaneously this model with compliance increases considerably the flexibility and universality of simulation tools in a wide range of applied problems.

\section{Conclusions}

Comparing our previous results with the above ones we can conclude that:

- since cylindrical contact models are restricted to the 2D-geometrical considerations they are simpler in a certain sense than the 3D-models;

- on the contrary, dynamical models became more complex in some degree because the Johnson model forces us to deal with the transcendental equation having a singularity at zero;
- involute meshing requires additional analytic efforts causing additional computational complexity increase;

- compliant models create an effect similar to one generated by the torsional elastic deformations of gearwheels;

- compliant model built up showed an efficiency high enough comparable with the fastest case of geometrically rigid constraint;

- the computer model built up makes it possible in an evident way to construct models of gearboxes of any complexity for the spur involute type of meshing.

\section{Acknowledgements}

The paper was prepared with partial support of Russian Foundation for Basic Research, projects 08-01- 
00600-a, 08-01-00718-a, 08-08-00553-a.

\section{References}

[1] Pelchen, C., Schweiger, C., Otter, M., Modeling and Simulating the Efficiency of Gearboxes and of Planetary Gearboxes. In: Proceedings of the 2nd International Modelica Conference, Deutsches Zentrum für Luft- und Raumfahrt e. V. (DLR), Oberpfaffenhofen, Germany, March 1819, 2002, pp. 257-266.

[2] Schlegel, C., Bross, M., Beater, P., HILSimulation of the Hydraulics and Mechanics of an Automatic Gearbox. In: Proceedings of the 2nd International Modelica Conference, Deutsches Zentrum für Luft- und Raumfahrt e. V. (DLR), Oberpfaffenhofen, Germany, March 1819, 2002, pp. 67-75.

[3] Schweiger, C., Otter, M., Modeling 3D Mechanical Effects of 1D Powertrains. In: Proceedings of the 3rd International Modelica Conference, Linköpings Universitet, Linköpings, Sweden, November 3-4, 2003, pp. 149-158.

[4] Kosenko, I. I., Loginova, M. S., Obraztsov, Ya. P., Stavrovskaya, M. S., Multibody Systems Dynamics: Modelica Implementation and Bond Graph Representation. In: Proceedings of the 5th International Modelica Conference, arsenal research, Vienna, Austria, September 4-5, 2006, pp. 213-223.

[5] Kosenko, I., Aleksandrov, E., Implementation of the Contensou-Erismann Model of Friction in Frame of the Hertz Contact Problem on Modelica. In: Proceedings of the 7th International Modelica Conference, Como, Italy, 2022 September 2009. Francesco Casella, editor. Linköping University Electronic Press, 2009. ISBN 978-91-7393-513-5. Linköping Electronic Conference Proceedings, ISSN:1650-3740. DOI: 10.3384/ecp0943, pp. 288-298.

[6] Kossenko, I. I., Implementation of Unilateral Multibody Dynamics on Modelica. In: Proceedings of the 4th International Modelica Conference, Hamburg University of Technology, Hamburg-Harburg, Germany, March 7-8, 2005, pp. 13-23.
[7] Kosenko, I. I., Alexandrov, E. B., Implementation of the Hertz Contact Model and Its Volumetric Modification on Modelica. In: Proceedings of the 6th International Modelica Conference, University of Applied Sciences Bielefeld, Bielefeld, Germany, March 3-4, 2008, pp. 203-212.

[8] Litvin, F. L., Fuentes, A., Gear Geometry and Applied Theory. Cambridge University Press, Cambridge - New York - Melbourne - Madrid - Cape Town - Singapore - Sao Paulo, 2004.

[9] Pereira, C. M., Ramalho, A. L., Ambrosio, J. A., A Critical Overview of Internal and External Cylinder Contact Force Models. Nonlinear Dynamics, Published Online: 18 September 2010.

[10] Johnson, K. L., Contact Mechanics. Cambridge University Press, Cambridge, UK, 2001.

[11] Förg, M., Engelhardt, T., Ulbrich, H. Comparison of Different Contact Models within Valve Train Simulations. Proceedings of ACMD2006, The Third Asian Conference on Multibody Dynamics 2006, Institute of Industrial Science, The University of Tokyo, Tokyo, Japan, August 1-4, 2006.

[12] Vaishya, M., Singh, R., Sliding Friction-Induced Non-Linearity and Parametric Effects in Gear Dynamics. Journal of Sound and Vibration, 2001, Vol. 248, No. 4, pp. 671-694.

[13] Vaishya, M., Singh, R., Strategies for Modeling Friction in Gear Dynamics. Journal of Mechanical Design, 2003, Vol. 125, Iss. 2, pp. 383-393.

[14] He, S., Effect of Sliding Friction on Spur and Helical Gear Dynamics and Vibro-Acoustics. PhD thesis, The Ohio State University, 2008. 